\title{
Hydrogen Storage Using Carbon Nanotubes
}

\author{
Yunjin Yao \\ Hefei University of Technology
}

China

\section{Introduction}

Hydrogen is the ideal candidate as an energy carrier for both mobile and stationary applications while averting adverse effects on the environment, and reducing dependence on imported oil for countries without natural resources. Hydrogen storage is clearly one of the key challenges in developing hydrogen economy. Hydrogen can be stored as (i) pressurized gas, (ii) cryogenic liquid, (iii) solid fuel as chemical or physical combination with materials, such as metal hydrides, complex hydrides and carbon materials, or produced on-board the vehicle by reforming methanol (Sakintuna, Lamari-Darkrim, \& Hirscher, 2007). Compressed and/or liquid hydrogen is currently used in fuel cell powered automobiles but application issues, such as safety and the need for a hydrogen supply infrastructure, are problems, which remain to be solved. Solid-state hydrogen storage systems are expected to be simpler for the engineering design of vehicles and considerably safer than the storage of elemental hydrogen (Fig. 1).

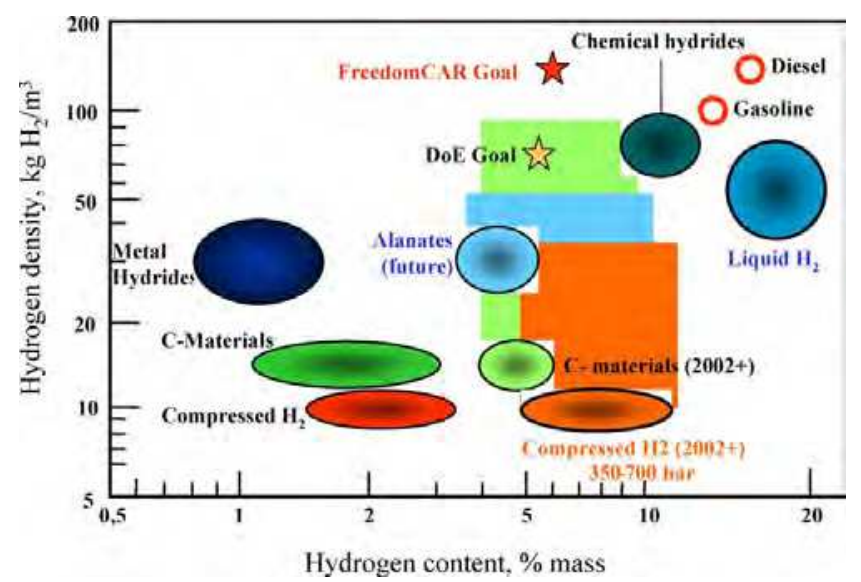

Fig. 1. Volumetric and gravimetric hydrogen density for different storage methods (R. Ströbel, J. Garche, P. T. Moseley, L. Jörissen, \& G. Wolf, 2006).

The US Department of Energy (DOE) published a long-term vision for hydrogen storage applications considering economic and environmental parameters. The predicted minimum 
hydrogen-storage capacity should be $6.5 \mathrm{wt} \%$ and $65 \mathrm{~g} / \mathrm{L}$ hydrogen available, at the decomposition temperature between 60 and $120{ }^{\circ} \mathrm{C}$ for commercial viability. It was also predicted low temperature of hydrogen desorption and low pressure of hydrogen absorption (a plateau pressure of the order of a few bars at room temperature) and nonthermal transformation between substrates and products of decomposition as reported by Schulz (Schulz et al., 1999). Furthermore, the cost of a storage medium and its toxicity properties need to be carefully considered for the realization of the set goals. The different time-dependent DOE targets are shown in Table 1.

\begin{tabular}{|l|l|l|l|}
\hline Storage parameter & 2005 & 2010 & 2015 \\
\hline Gravimetric capacity (specific energy) $\left(\mathrm{kW} \mathrm{h} \mathrm{kg}^{1}\right)$ & 1.5 & 2.0 & 3.0 \\
\hline Gravimetric capacity (specific energy) $\left(\mathrm{kgH}_{2} \mathrm{~kg}^{1}\right)$ & 0.045 & 0.060 & 0.090 \\
\hline System weight $(\mathrm{kg})$ & 111 & 83 & 55.6 \\
\hline Volumetric capacity (energy density) $\left(\mathrm{kW} \mathrm{h} \mathrm{l-1}^{-1}\right)$ & 1.2 & 1.5 & 2.7 \\
\hline Volumetric capacity (energy density) $\left(\mathrm{kgH}_{2} \mathrm{l}^{-1}\right)$ & 0.036 & 0.045 & 0.081 \\
\hline System volume $(\mathrm{l})$ & 139 & 111 & 62 \\
\hline Storage system cost $\left(\mathrm{US}^{\mathrm{k}} \mathrm{kW} \mathrm{h}^{-1}\right)$ & 6 & 4 & 2 \\
\hline System cost $(\mathrm{US} \$)$ & 1000 & 666 & 333 \\
\hline Refueling rate $\left(\mathrm{kgH}_{2} \mathrm{~min}^{-1}\right)$ & 0.5 & 1.5 & 2.0 \\
\hline Refueling time $(\mathrm{min})$ & 10 & 3.3 & 2.5 \\
\hline
\end{tabular}

Table 1. DOE targets for hydrogen on-board storage and specific data for a $5 \mathrm{kgH}_{2}$ system

Storage by absorption as chemical compounds or by adsorption on carbon materials have definite advantages from the safety perspective such that some form of conversion or energy input is required to release the hydrogen for use. A great deal of effort has been made on new hydrogen-storage systems, including metal, chemical or complex hydrides and carbon nanostructures. Carbon materials such as activated carbons, carbon nanotubes, and carbon nanofibers have been the subject of intensive research. The research on hydrogen storage in carbon materials was dominated by announcements of high storage capacities in carbon nanostructures.

However, the experimental results on hydrogen storage in carbon nanomaterials scatter over several orders of magnitude. The hydrogen-storage capacity for carbon materials is reported between 0.2 and $10 \mathrm{wt} \%$. The experiments to date claiming very high values could not independently be reproduced in different laboratories. In view of today' s knowledge although they have good reversibility properties, carbon nanostructures cannot store the amount of hydrogen required for automotive applications.

This Chapter deals with hydrogen adsorption in carbon nanotube materials over a wide range of pressure and temperature in order to establish the reliability of this phenomenon as a valuable storage technique of hydrogen. Our purpose is to give, discuss and compare the different results published comprising our works. Both experimental and theoretical investigations data concerning the hydrogen gas within nanotube material framework are given. A comparison between the different studies should enable to gain a better knowledge of hydrogen adsorption in nanotubes and then to answer the following questions: How and where did the gas adsorption occur? Moreover, we will consider the possibility of controlling tube diameters and lengths that will enable to optimize nanotube structure for a 
maximal hydrogen adsorption. Indeed, the possibility of controlling microscopic parameters will enable to control the main material macroscopic property: the gas adsorption. Finally, it is noticeable that both the material synthesis and purification will have to be optimized in order to enable the gas adsorption measurements. Indeed, the adsorbent material will have to be available in large amounts with high degree of purity to ensure the hydrogen storage by adsorption in carbon nanotubes

\section{Hydrogen storage fundamentals}

\subsection{Physisorption}

Physisorption is based on the van der Waals interaction (a resonant fluctuation in the charge distribution) between gases and solids. These are attractive forces, which keep graphene layers well-stacked, and are the same intermolecular forces that play an important role in the physisorption mechanism of gasses and hydrogen on carbon surfaces. Van der Walls bonds stem from intermolecular forces between atoms (and/or molecules) that result from instantaneous charge distribution in atoms and molecules when they approach each other. Under mutual interaction, an asymmetric polarization of electron charge is induced in molecules that create temporarily dipole moments, and atoms or molecules become attracted by electrostatic forces (Fig. 2).The interaction energy, also called the London Dispersion forces $\left(E_{S-H_{2}}\right)$, between the substrate $(S)$ and the hydrogen molecule $\left(\mathrm{H}_{2}\right)$, is given by

$$
E_{S-H_{2}} \sim \frac{\alpha_{H_{2}} \alpha_{S}}{R^{6}}
$$

where $\alpha$ is the polarizibility and $\mathrm{R}$ is the interaction distance. Because $\alpha_{\mathrm{H}_{2}}$ is fixed, the only way to increase $E_{{\mathrm{S}-\mathrm{H}_{2}}_{2}}$ is to use highly polarizable substrates, e.g. substrates with ח-electron systems.
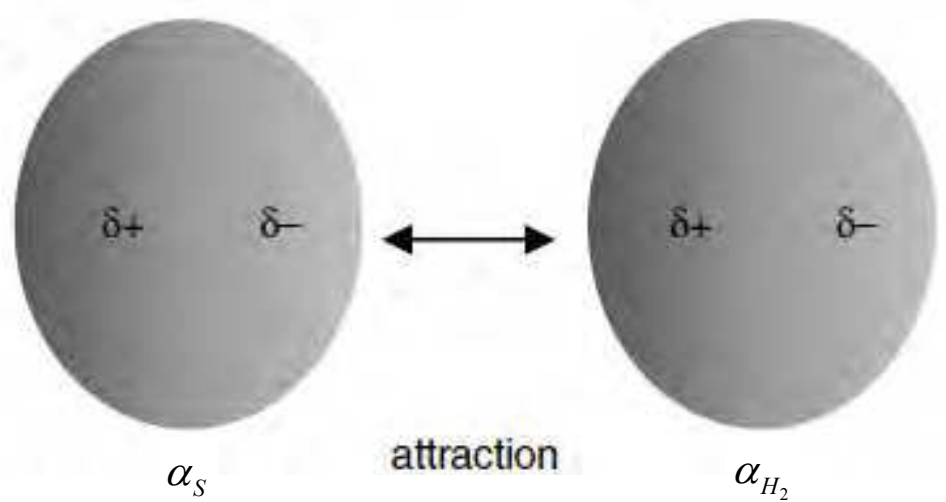

Fig. 2. Van der Walls bonding as originating from the polarizibility of electron density on hydrogen molecule and carbon atom

The equilibrium between gas attraction and gas repulsion on the surface creates an energy minimum which amounts to between 1 and $10 \mathrm{~kJ} \mathrm{~mol}^{-1}$. The exact value for the hydrogen 
adsorption on a flat carbon surface depends on the adsorption stereometry. An average value would be about $4-5 \mathrm{~kJ} \mathrm{~mol}^{-1}$. This represents a very weak interaction. Therefore, hydrogen is desorbed with increasing temperature, and very little hydrogen adsorption is observed on carbon at elevated temperatures(R. Ströbel, J. Garche, P. Moseley, L. Jörissen, \& G. Wolf, 2006).

Normally only a monolayer is adsorbed above the boiling point of the adsorbent. In this case the Langmuir isotherm should apply. The maximum value of hydrogen adsorption on any particular material can be estimated as the quantity of hydrogen that can be accommodated in a monolayer. With the assumption that the structure of the adsorbed hydrogen is closedpacked face centered, the minimum surface area for the adsorption of 1 mol of hydrogen amounts to $\mathrm{Sml}\left(\mathrm{H}_{2}\right)=85.917 \mathrm{~m}^{2} \mathrm{~mol}^{-1}$. Based on the surface area of a single graphene sheet $\left(1315 \mathrm{~m}^{2} \mathrm{~g}^{-1}\right)$ the maximum value for the storage capacity of hydrogen adsorbed on graphite is calculated to be about $3 \mathrm{wt} \%$. This value, however, can be reached only at very low temperatures. Based on this approximation the theoretical hydrogen storage capacity (mads) can be calculated from the specific surface of the carbon $\left(S_{\text {spec }}\right)$ : mads $\approx S_{\text {spec }} \times 2.27 \times 10^{-3} \mathrm{wt} \%$; the amount of adsorbed hydrogen correlates with the specific surface of carbon. Taking this equation into account a theoretical carbon surface area of $2600 \mathrm{~m}^{2} \mathrm{~g}^{-1}$ would be necessary to meet the 2010 DOE target (6 wt \%).

Fig. 3 shows the experimental relationship between different carbon surfaces and the hydrogen storage capacity under different measurement conditions.An extrapolation shows that the 2010 DOE target ( $6 \mathrm{wt} \%$ ) would be reached at a carbon surface of $>4000 \mathrm{~m}^{2} \mathrm{~g}^{-1}$. This is not yet available.

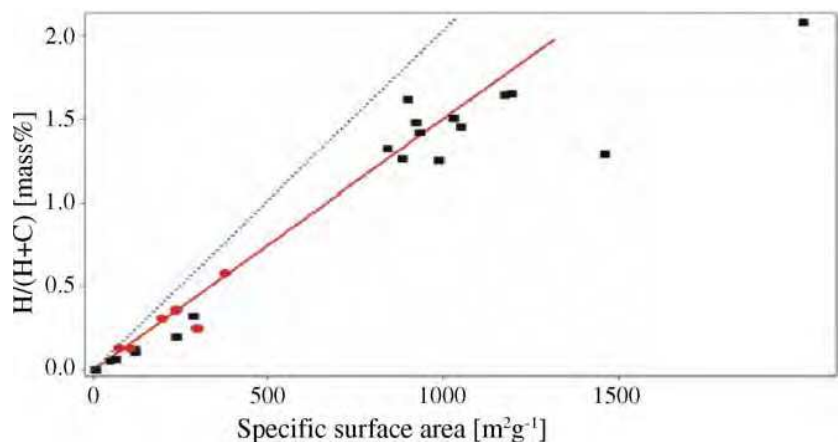

Fig. 3. Reversible amounts of hydrogen adsorbed vs. surface area (Züttel, 2003). (Circles) Carbon nanotubes, high surface area graphite (electrochemical measurement at $298 \mathrm{~K}$ ). (Squares) Hydrogen adsorption measurement at 77K from Nijkamp et al. (Nijkamp, Raaymakers, Van Dillen, \& De Jong, 2001). (Dotted line) Calculated amount of hydrogen in a monolayer at the substrate surface.

\subsection{Chemisorption}

If the $\pi$-bonding between carbon atoms were to be fully utilized, every carbon atom could be a site for chemisorption of one hydrogen atom. Desorption results of nanotubes treated with hydrogen under high pressure show a peak at temperatures above $400 \mathrm{~K}$, which indicates chemisorption. This is ascribed to a completelayer of chemisorbed hydrogen on the outside wall of the nanotube. A first-principle calculation shows that a dissociative chemisorption of 
hydrogen on carbon nanotubes should be possible. The H-H bond breaking (bonding energy $4.52 \mathrm{eV}$ ) is concerned with the formation of two $\mathrm{C}-\mathrm{H}$ bonds on two adjacent nanotubes in the solid phase, and is facilitated by the application of high pressure, which shortens the distance between nanotubes from a distance of $1.2 \mathrm{~nm}$ at atmospheric pressure to about1.05 $\mathrm{nm}$ at high pressure. This leads to a shortening of the C-C separation between two adjacent tubes from 3.8 to $2.6 \AA$, and this facilitates dissociative hydrogen adsorption. The chemisorbed hydrogen, however, can only be released at higher temperatures. Thus, chemisorption is not useful for the practical storage of hydrogen.

Hydrogen storage in CNTs by chemical reaction, on the other hand, has largely been discounted as irreversible and thus technologically less relevant. Whereas the release of chemically stored hydrogen is not as facile as that of physisorbed hydrogen, given the controversy of the latter, the topic deserves careful consideration for several reasons. First, the reproducible experiments of Liu et al(C. Liu et al., 1999). demonstrate that, of the $4.2 \mathrm{wt}$ $\%$ hydrogen that is adsorbed at moderate pressures and room temperature, $78.3 \%$ (3.3 wt \%) is physisorbed, whereas the extraction of residual stored hydrogen $(0.9 \mathrm{wt} \%)$ requires heating to $473 \mathrm{~K}$ and above. This suggests that some form of chemical adsorption occurs at room temperature. Second, it has been shown that CNTs are capable of storing a modest amount of hydrogen electrochemically. Third, under a high applied pressure of hydrogen, a first-order phase transformation in CNTs has been reported, which is reminiscent of hydride phases in metal-hydrogen systems. It was demonstrated recently that the high-pressure hydrogenation of SWNTs results in $\mathrm{H}$ covalently bound to $\mathrm{C}$ at $4.7 \mathrm{wt} \%$ and interstitial $\mathrm{H}_{2}$ at $0.5 \mathrm{wt} \%$. In addition, novel procedures using hydrogen plasma 15-18 and polyamine reagents19 have been harnessed to achieve a sizable degree of chemical hydrogenation in SWNTs. Finally, a highly nonequilibrium method of CNTs hydrogenation by the implantation of molecular and atomic hydrogen beams has been proposed.9,20 Recent experimental studies have demonstrated that a high degree of hydrogenation of SWNTs, 65 ( $15 \%$ (5.1 ( $1.2 \mathrm{wt} \%)$, can be achieved with atomic $\mathrm{H}$ beams and that, just as importantly, the process is reversible(Bilic \& Gale, 2008).

\subsection{Adsorption energy}

An important parameter for the characterization of hydrogen storage is the adsorption energy, $\Delta E(a d s)$.If the adsorption energy is low, i.e. we have a weak interaction between hydrogen and carbon, hydrogen will desorb spontaneously at low temperatures. Then hydrogen storage at ambient temperature would only be possible at high pressure. If the adsorption energy is high, a relative large amount of hydrogen could be adsorbed, but the adsorbed hydrogen would not readily desorb, and only a small amount of fuel would be available.

It would be useful to be able to design a sorbent that adsorbs strongly but whose interaction potential could be attenuated when hydrogen was to be desorbed. Charged carbon nanotubes could form such a system. The charge-quadrupole interaction between the substrate and the quadrupolar hydrogen molecule is attractive and is longer ranged than the dispersive forces. By controlling the charge of the sorbent, as in the case of capacitors, hydrogen could be encouraged to adsorb or desorb, as and when required. An increase of about $30 \%$ in the amount of hydrogen adsorbed on charged SWNTs compared with uncharged SWNTs sorbents has been observed for realistic chemical charges of $0.1 \mathrm{e} \mathrm{atom}^{-1}$. The activation energies of the adsorption $\left(E_{a}^{\text {ads }}\right)$ and desorption $\left(E_{a}^{\text {des }}\right)$ processes play an important role in system kinetics. There is no barrier to be overcome in the adsorption 
process, i.e. $E_{a}^{a d s}=0$. However, there is a significant barrier for the desorption process. To a first approximation, the magnitudes of the energy of adsorption and the activation energy for desorption are equal; $E_{a}^{a d s} \approx \Delta E(a d s)$.

The adsorption energy of different hydrogen storage materials is shown in Fig. 4. The favorable binding energy is in the region of $10-50 \mathrm{~kJ}$ mol-1, because these values are acceptable for room temperature storage. Such values could be reached by carbon materials with:

(i) Enhanced physisorption: An enhanced physisorption takes place if the interlayer distance between graphene layers is optimized by spacers. The additives act not only geometrically but also via a strong interaction with hydrogen. This interaction can increase the adsorption energy leading to a higher desorption temperature. However, this advantage must be weighed against the increase of the material density.

(ii) 'Kubas' binding: Kubas (Kubas, 2001) discovered molecular hydrogen complexes of transition metals. In these complexes, mostly organometal complexes, the chemical bond in the hydrogen molecule interacts with metals and leads to a relatively strong adsorption of molecular hydrogen. This interaction is due to donation of charge from the highest occupied orbital of the ligand $\left(\mathrm{H}_{2}\right)$ to the empty metal orbitals and a subsequent back-donation from filled dorbitals into the lowest unoccupied orbital of the ligand.

(iii) 'Spillover': Additives act as a catalytic active center for the dissociation of hydrogen. The dissociated hydrogen atom then can spillover from the additive sites to the carbon network and finally become bound to carbon atoms. This has been described, e.g. by Chen et al. for Li-doped MWNTs (P. Chen, X. Wu, J. Lin, \& K. L. Tan, 1999).

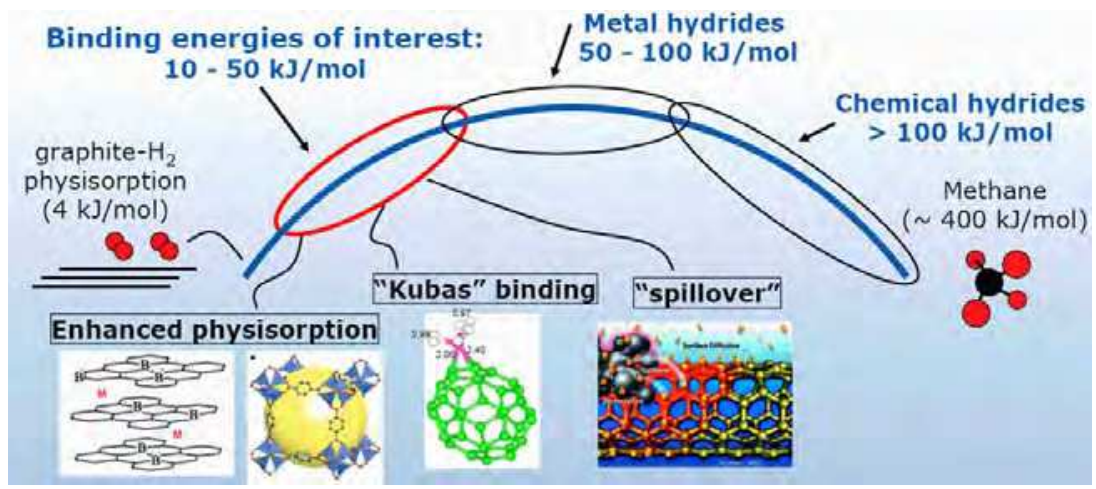

Fig. 4. Overview of the binding energies of different hydrogen adsorption materials

\section{Practical adsorption storage of hydrogen with carbon materials}

\subsection{Single-walled nanotubes (SWNTs)}

In 1997, Dillon et al. (A. C. Dillon et al., 1997) first published investigations on the hydrogen storage capacity of single-walled carbon nanotubes. Their experimental procedure, which they named Temperature Programmed Desorption, consists in measuring with a mass spectrometer the hydrogen desorbed by the carbon sample during a heating run. Nonpurified samples containing only a trace of SWNTs were exposed to hydrogen at ambient pressure and room temperature. The amount of hydrogen in the overall sample was 
measured at $0.01 \mathrm{wt} \%$ by thermal desorption spectroscopy. Based on this measurement the authors estimated a hydrogen storage capacity of $5-10 \mathrm{wt} \%$ for pure SWNTs, assuming that the small hydrogen content was due to the small fraction of SWNTs present in the sample. In further work, Dillon et al. developed a special opening and cutting procedure and measured a storage capacity of about $7 \mathrm{wt} \%$ for purified SWNTs after loading at $0.07 \mathrm{MPa}$ and at room temperature by prior heating to $900 \mathrm{~K}$ in high vacuum. However, the temperature of hydrogen release was now significantly higher double maximum at 450 and $600 \mathrm{~K}$ ) compared to their former investigations (single maximum at $300 \mathrm{~K}$ ). The opening and cutting method was high-power ultra-sonica- tion in $\mathrm{HNO}_{3}$, which results in contamination of the sample with metal particles due to cavitation of the sonicator horn [8]. The material of this horn has the composition $90 \mathrm{wt} \% \mathrm{Ti}, 6 \mathrm{wt} \% \mathrm{Al}$, and $4 \mathrm{wt} \% \mathrm{~V}$, which is commonly used because of its chemical resistance. The authors proposed that the introduced Ti alloy particles 'somehow' stimulates hydrogen adsorption of the SWNTs.

Liu et al. (C. Liu et al., 1999)determined the adsorption of hydrogen on SWNTs having a purity of $50-60 \%$ which they have synthesized by a semi-continuous hydrogen arc discharge technique. They also treated their samples by chlorhydric acid and vacuum heated at high temperature and compared the corresponding incidence of these purifying treatments. Their adsorption measurement technique consists in monitoring versus time the pressure change of hydrogen introduced in a constant-volume cell containing the nanotubes sample; when the equilibrium is achieved the total decrease of pressure corresponds to the amount of hydrogen adsorbed. The results are, respectively, $2 \% ; 2.5 \%$ and $4 \%$ weight for non-treated, partially treated and entirely treated samples. As concerns the two thermal effects resulting from the hydrogen milling of the sample cell, they have apparently not been taken into account. One of these effects is due to the gas compression up to the target filling pressure, $10-12 \mathrm{MPa}$; the second is due to the gas adsorption during this 9lling. The two thermal contributions being exothermic, they contribute to increase the gas temperature and then the gas pressure at the initial time of the pressure change measurements with the consequence of overestimating the amount of hydrogen adsorbed.

Ye et al. (Y Ye et al., 1999) reported that a ratio of $\mathrm{H}$ to $\mathrm{C}$ atoms of about 1.0 was obtained for crystalline ropes of SWNTs at $80 \mathrm{~K}$ and pressures.12 MPa. At a pressure of $4 \mathrm{MPa}$, a sudden increase in the adsorption capacities of the SWNTs samples was reported, and they suspect that a structural phase transition is responsible for this effect.

In Fig. 5, the optical absorption spectrum of purified SWNTs thin films measured by Kataura et al. (Kataura et al., 1999) is presented. The three peaks at $0.68,1.2$ and $1.7 \mathrm{eV}$ were found to correspond to the DOS peaks of two semiconducting and one metallic tubes. Additionally, the large absorption band at $4.5 \mathrm{eV}$ was ascribed to the p-plasmon of SWNTs observed also in the energy-loss spectrum. On doping with electron donors (K, Cs) or acceptors $\left(\mathrm{Br}_{2}, \mathrm{I}_{2}\right)$, the absorption bands of SWNTs are smoothed and completely vanish at high doping level $(x>0: 04)$. This phenomenon is explained by charge transfer from the donors to the nanotubes, filling up the lowest unoccupied bands and depleting the absorption bands. In a similar way, the acceptors lead to depletion of absorption bands. Hwang et al. (Hwang et al., 2000)measured the reflectance of an unaligned mat of SWNTs in the range $0.003-3 \mathrm{eV}$. The reflectance spectrum exhibited peaks at $0.7,1.3,1.9$, and $2.6 \mathrm{eV}$ ascribed to transitions between the DOS peaks of semiconducting and metallic nanotubes. The polarized absorption of a freestanding SWNTs fiber showed considerable anisotropy with bands at 1.9 and $2.6 \mathrm{eV}$ being strongly polarized in parallel direction(Popov, 2004). 


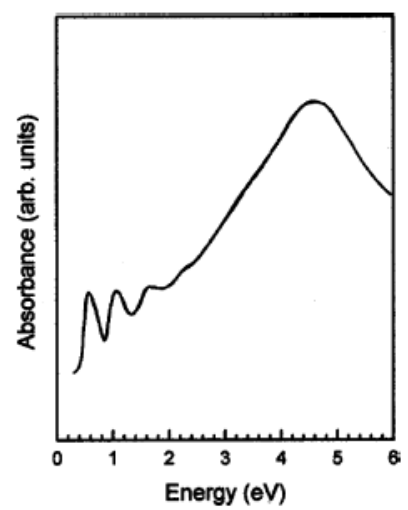

Fig. 5. Optical absorption of purified SWNTs thin film samples synthesized by the arcdischarge method using a NiY catalyst (after (Kataura et al., 1999)).

\subsection{Multi-walled nanotubes (MWNTs)}

Multi-wall carbon nanotubes consist of layers of nested concentric cylinders of graphite with a hollow center.The adsorption properties of this type of nanotubes has been recently studied.

Chen et al(C. Chen \& Huang, 2007). reported remarkable hydrogen-storage capacities for alkali-metal-doped MWNTs formed by the catalytic decomposition of $\mathrm{CH}_{4}$. Experimental results revealed that the structure of carbon nanotubes (CNTs) became destructive after being activated by $\mathrm{KOH}$ at $823 \mathrm{~K}$ in $\mathrm{H}_{2}$ atmosphere. It was also found that the capacity of hydrogen storage onto the unmodified and $\mathrm{KOH}$ modified CNTs were 0.71 and $4.47 \mathrm{wt} \%$, respectively, under ambient pressure and at moderate temperature. In addition to alkali metal, both the residual metal such as cobalt and the defect structure were also considered as important keys to be used to improve hydrogen adsorption in the CNTs. The cause of which suggested that residual metal such as cobalt may be acting to dissociate hydrogen molecule into its atom state to spread out to the surface and inner layer through defect sites of the CNTs.

A very recent report probed the hydrogen-adsorption properties of multi-wall nanotubes synthesized by the catalytic decomposition of $\mathrm{CO}$ and $\mathrm{CH}_{4}$ on powdered $\mathrm{La}_{2} \mathrm{O}_{3}$ catalysts $(\mathrm{Wu}, \mathrm{Chen}, \mathrm{Lin}, \&$ Tan, 2000). The CO-generated tubes consisted of concentric cylinders while the $\mathrm{CH}_{4}$-produced tubes contained graphite layers that were tilted with respect to the tube axis, forming cones. In both cases the catalyst was removed by stirring in dilute nitric acid. The purified nanotubes were then annealed to $1100^{\circ} \mathrm{C}$ in vacuum in order to increase crystallinity. TGA analysis in flowing hydrogen revealed that the CO-generated tubes were capable of adsorbing a small quantity of hydrogen $(0.25 \mathrm{wt} \%)$ when the sample was cooled between $200^{\circ} \mathrm{C}$ and room temperature. Another recent study indicated that MWNTs may also be charged with hydrogen by electrochemical methods (Nützenadel, Züttel, Chartouni, \& Schlapbach, 1999). Materials employed for this study were synthesized in an arc process and contained $1040 \%$ MWNTs with diameters which varied from 2 to 15 $\mathrm{nm}$. Stable electrodes were be formed by pressing the MWNTs material with palladium powder in a 1: 4 ratio. The equilibrium curve of the MWNTs/palladium electrode indicated 
two separate electrochemical reactions, one of which was not observed for pure palladium electrodes. Although the overall capacity for the palladium/MWNTs electrode was less than that anticipated for pure palladium, the study indicated that electrochemical hydrogen storage in MWNTs may be possible (A. Dillon \& Heben, 2001).

\subsection{Analysis of the results}

An overview of experimental data on hydrogen adsorption in carbon nanotubes is given in the literature by Ding et al. Table 2 shows hydrogen storage values between 0.05 and $21 \mathrm{wt} \%$. The high values, however, have not so far been verified independently.

The storage values are dependent on many parameters of the carbon nanotubes, including their structure, structure defects, pretreatment, purification, geometry (surface area, tube diameter, and length), arrangement of tubes in bundles and/or 'ropes', storage pressure, temperature, etc.

Hydrogen uptake varies linearly with tube diameter even though the tube volume increases as the square of the radius. This is because the uptake is proportional to the surface area (one layer adsorption), i.e. the number of carbon atoms, which increases linearly with increasing tube diameter. The tube diameter can be controlled via the synthesis conditions. No complete agreement exists about the adsorption sites in carbon nanotubes. Theoretically the following sites exist:

- inside the tube;

- outside the tube;

- between tubes in 'bundles' and 'ropes';

- between the shells in multi-walled nanotubes.

For carbon nanotube bundles the adsorption sites are shown schematically in Fig. 6. For hydrogen storage within the tube the hydrogen either must pass through the CNTs wall or the tube must be open.
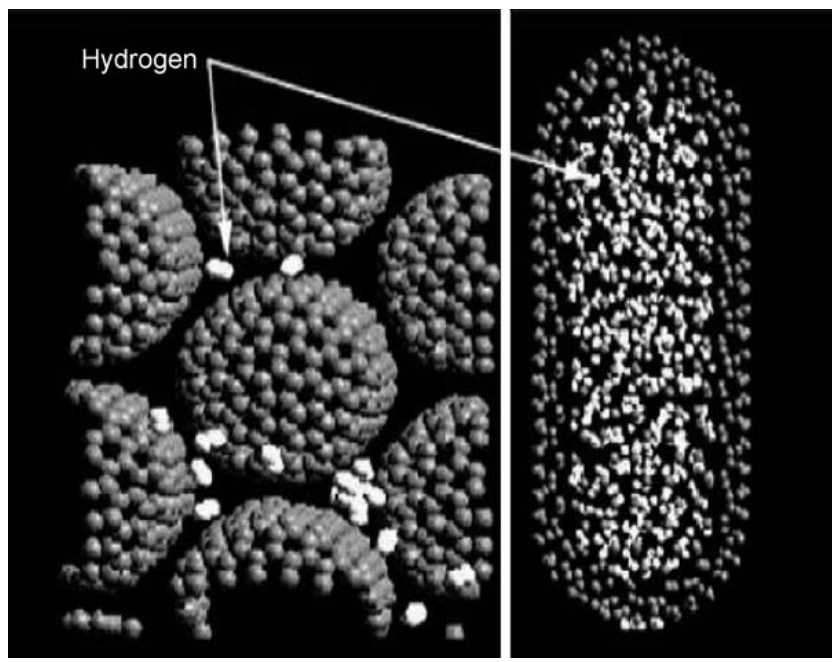

Fig. 6. Potential sites for hydrogen adsorption within a nanotube bundle; (left) interstitial sites between tubes and (right) inside the tube . 


\begin{tabular}{|c|c|c|c|c|c|}
\hline Sample & Purity (\%) & $\mathrm{T}(\mathrm{K})$ & $\mathrm{P}(\mathrm{MPa})$ & $\mathrm{H}_{2}(\mathrm{wt} \%)$ & Ref. \\
\hline SWNTs & Assumed 100 & 133 & 0.04 & $5-10$ & (A. C. Dillon et al., 1997) \\
\hline SWNTs & 50 & 300 & 10.1 & 4.2 & (C. Liu et al., 1999) \\
\hline Aligned SWNTs & Purified & Ambient & 11 & 4 & $\begin{array}{l}\text { (Liu, Yang, Tong, Cong, \& } \\
\text { Cheng, 2002) }\end{array}$ \\
\hline SWNTs & High & 80 & 7 & 8.25 & (Y. Ye et al., 1999) \\
\hline MWNTs & Purified & $\sim 300-700$ & Ambient & 0.25 & (Wu et al., 2000) \\
\hline SWNTs & Purified & Ambient & 4.8 & 1.2 & $\begin{array}{l}\text { (Smith Jr, Bittner, Shi, } \\
\text { Johnson, \& Bockrath, 2003) }\end{array}$ \\
\hline SWNTs-Ti-6Al-4V & Purified & Ambient & 0.08 & 1.7 & (Hirscher et al., 2001) \\
\hline SWNTs-Fe & Purified & Ambient & 0.08 & $<0.005$ & (Hirscher et al., 2001) \\
\hline $\begin{array}{l}\text { Ball-milled SWNTs } \\
\text { in Ar }\end{array}$ & $<50$ & Ambient & 0.08 & $<0.1$ & (Hirscher et al., 2002) \\
\hline $\begin{array}{l}\text { Ball-milled SWNTs } \\
\text { in D2 }\end{array}$ & $<50$ & Ambient & 0.9 & 0 & (Hirscher et al., 2002) \\
\hline CNTs & Purified & $298-773$ & 0.1 & 0 & (Hirscher et al., 2002) \\
\hline Li-CNTs & Purified & $473-673$ & 0.1 & 20 & $\begin{array}{l}\text { (P. Chen, X. Wu, J. Lin, \& } \\
\text { K. Tan, 1999) }\end{array}$ \\
\hline K-CNTs & Purified & $<313$ & 0.1 & 14 & (P Chen et al., 1999) \\
\hline Li-CNTs (wet $\mathrm{H}_{2}$ ) & Purified & $473-673$ & 0.1 & 12 & (Yang, 2000) \\
\hline Li-CNTs (dry $\left.\mathrm{H}_{2}\right)$ & Purified & $473-673$ & 0.1 & 2.5 & (Yang, 2000) \\
\hline K-CNTs (wet $\mathrm{H}_{2}$ ) & Purified & $<313$ & 0.1 & 21 & (Yang, 2000) \\
\hline K-CNTs (dry $\mathrm{H}_{2}$ ) & Purified & $<313$ & 0.1 & 1.8 & (Yang, 2000) \\
\hline Li-CNTs & $10-40$ & $473-663$ & 0.1 & $0.7-4.2$ & (Pinkerton et al., 2000) \\
\hline SWNTs & $90 \mathrm{vol} \%$ & 298 & - & 0.63 & (Ritschel et al., 2002) \\
\hline MWNTs & Unpurified & 298 & - & 0.5 & (Ritschel et al., 2002) \\
\hline CNTs & - & Ambient & Ambient & 0.5 & $\begin{array}{l}\text { (Adu, Sumanasekera, } \\
\text { Pradhan, Romero, \& } \\
\text { Eklund, 2001) }\end{array}$ \\
\hline SWNTs & Purified & 77 & 0.2 & 6 & (Pradhan et al., 2002) \\
\hline Aligned MWNTs & High & 298 & 10 & 3 & (Zhu et al., 2001) \\
\hline Aligned MWNTs & High & 290 & 10 & 2.4 & (Cao et al., 2001) \\
\hline Random MWNTs & High & 298 & 10 & 0.68 & (Zhu et al., 2001) \\
\hline MWNTs & High untreated & 300 & 1.0 & $5-7$ & (Y. Chen et al., 2001) \\
\hline MWNTs & $\begin{array}{l}\text { High acid } \\
\text { treated }\end{array}$ & 300 & 1.0 & 13.8 & (Y. Chen et al., 2001) \\
\hline MWNTs & High & 300 & 7.0 & $0.7-0.8$ & $\begin{array}{l}\text { (Badzian, Badzian, Breval, } \\
\text { \& Piotrowski, 2001) }\end{array}$ \\
\hline SWNTs & Unpurified & 295 & 0.1 & 0.93 & (Nishimiya et al., 2002) \\
\hline SWNTs & Unpurified & 77 & 0.1 & 2.37 & (Nishimiya et al., 2002) \\
\hline MER MWNTs & $10-15 \%$ & 298 & 3.6 & 0.03 & $\begin{array}{l}\text { (Tibbetts, Meisner, \& Olk, } \\
\text { 2001) }\end{array}$ \\
\hline Rice SWNTs & High & 298 & 3.6 & 0.05 & (Tibbetts et al., 2001) \\
\hline CNTs & - & 298 & 11.35 & 11.26 & $\begin{array}{l}\text { (Chambers, Park, Baker, \& } \\
\text { Rodriguez, 1998) }\end{array}$ \\
\hline
\end{tabular}

Table 2. Summary of the reports of gaseous hydrogen storage capacity in carbon nanotubes

As shown in both Tables 2 extremely high hydrogen storage values (up to $21 \mathrm{wt} \%$ for the KCNTs structure) have been reported. As already mentioned a worldwide effort to verify these large hydrogen storage values in various nanostructured carbons, however, has not 
been successful. It is surprising, therefore, that very often these values continue to be cited in the literature without any acknowledgement of their uncertainty. The reasons why confirmation of these results has not been forthcoming could include the following:

- Low purity of samples: Syntheses of carbon nanostructured samples, especially those formed shortly after the discovery of these structures, tended to produce rather impure phases. Very often only small fractions of carbon nanotubes were found in the products and the hydrogen storage capacity of those samples was extrapolated in order to predict the capacity of pure materials.

- Insufficient characterization of samples: The carbon samples are very often a mixture of single-walled nanotubes, multi-walled nanotubes, and carbon nanofibers with various diameters, lengths, chiral vectors (in the case of nanotubes), shell numbers (in the case of multi-walled tubes), open and closed structures (tubes), etc. Because of all this potential variability it would not be surprising to find a large range of hydrogen storage values.

- Contamination of samples: For example, a storage capacity of about $4.5 \mathrm{wt} \%$ of hydrogen was found for single-walled nanotubes at $30 \mathrm{kPa}$ and $70 \mathrm{~K}$. Only a few milligrams of SWNTs were used for these investigations and the tubes were opened by a high power ultrasonic procedure in nitric acid(A. C. Dillon et al., 1997). The ultrasonic horn that was used was made from a corrosion-resistant $\mathrm{Ti}-6 \mathrm{Al}-4 \mathrm{~V}$ alloy. This alloy, however, is also a hydrogen storage material. Hirscher et al. (Hirscher et al., 2001) have shown that nearly all of the storage capacity of the sonicated samples examined in this work can be attributed to the hydrogen uptake of titanium alloy particles which have contaminated the carbon samples during sonication.

- Hydrogen contamination: Chen et al. (C Liu et al., 1999) found hydrogen storage capacities up to $20 \mathrm{wt} \%$ for lithium-doped multi-walled nanotubes and up to $14 \mathrm{wt} \%$ for lithium-doped graphite. It was shown, however, that the majority of the weight changes in this case are caused by adsorbed water, which was present as a contaminant in the hydrogen. Furthermore the $\mathrm{Li}_{2} \mathrm{O}$ used for the carbon treatment is both hygroscopic and able to adsorb carbon dioxide. Both of these processes lead to weight gains that could be mistakenly attributed to hydrogen uptake.

- Technical problems: The relatively complicated methods of synthesis of these materials often result in the production of only a rather small mass of carbon nanotubes and/or nanofibers. There is thus considerable scope for errors during gravimetric or volumetric measurement. In addition, inaccurate cooling of the hydrogen gas after pressurization can lead to effects that simulate adsorption. Problems can also arise through leaks in measurement equipment.

\section{Simulations of hydrogen adsorption in carbon nanotubes}

A number of theoretical investigations have been performed. These can be classified in two different groups. On the one hand, there are the grand canonical Monte Carlo simulations, which regard the uptake as a simple physical adsorption process. Their aim is to determine the adsorption isotherms on the assumption that no chemical process takes place and that the interaction between the carbonaceous materials and the hydrogen molecules can be described via a classical empirical potential. On the other hand, there is evidence that during the uptake a chemical process does indeed take place. This is also suggested by chemical intuition. The simulation of these processes requires that the electronic states are explicitly 
simulated. So far only two such sets of calculations have been performed by Lee et al. (S. Lee, An, Lee, Seifert, \& Frauenheim, 2001a), who studied the chemisorption process in nanotubes. calculations suggest that chemical processes do take place and that the hydrogen uptake is more complex than previously anticipated.

\subsection{Classical calculations (physisorption)}

Many scientific publications are devoted to the theoretical study of physical adsorption of gases on different adsorbent structures. Among these, noble gases are widely studied due to the inertness of these gases near reactive surfaces such as graphite planes and carbon nanotubes walls. These works concern both theoretical and realistic models of molecular gases in pores described by hard walls or attractive interfaces. They established that the physical adsorption of gases on a solid adsorbent can be accurately computed by numerical simulation. However, concerning carbon nanotube a few experimental works have been devoted to experimental measurements of gas adsorption in these adsorbents. The adsorption of diatomic gases such as hydrogen and nitrogen has been studied at different pressures and temperatures with the aim of investigating the physical mechanism of adsorption in carbon nanotubes. The agreement between simulation and experimental results indicates that the potential of interaction of carbon nanotubes is well described by the current potential used for planar graphite surfaces. Moreover, it is noticeable that the possibility of controlling microscopic parameters such as the nanotube diameters and lengths will enable to control material macroscopic properties such as gas adsorption. In the case of hydrogen adsorption, the storage of the gas in nanoscale-dimension material seems to be a good opportunity(FL Darkrim, Malbrunot, \& Tartaglia, 2002).

Wang and Johnson have also used the Feynmann path integral in order to account for the quantum effects of hydrogen (Wang \& Johnson, 1999). The results of these calculations show that the highest possible adsorption is achieved in nanofibers with large interlayer distance at $77 \mathrm{~K}$. While it is not clear whether such large interplanar distances can be achieved, it is discouraging to note that the storage capacity decreases very rapidly with increasing temperature. In the calculations that involve the arrays of nanotubes, the effect of change in geometry has been studied in some detail. Change in geometry can result both from changes in internal geometry (e.g. diameter) of the nanotubes and from the the relative arrangement of the nanotubes. As usual the geometry of a single nanotube is determined by two integer numbers that describe the way in which the graphite sheets are wrapped to form the nanotubes. The two-dimensional geometrical arrangement of nanotube arrays is expressed by the van der Waals gap, $g$, which measures the available interstitial space. $g$ is defined as $g=a D$, where $a$ is the lattice spacing and $D$ is the diameter of the nanotubes. The results do not show encouraging storage capacities at any temperature or pressure(Meregalli \& Parrinello, 2001).

Indeed, Darkrimand Levesque (Farida Darkrim \& Levesque, 2000) in recent numerical simulations have shown that adsorbent materials appropriate for hydrogen storage could be made up of carbon nanotubes constituted by nanoscale-dimension pores. Monte-Carlo numerical simulation based upon equilibrium statistical mechanics corresponds to a thermodynamic evaluation of the amount of gas adsorbed near the adsorbent surface. To compute this calculation, gas-adsorbent and gas-gas molecular interaction data are needed. Wang and Johnson (Wang \& Johnson, 1999)have presented different works on hydrogen 
and xenon adsorption in nanotube materials both at room temperature and at liquid nitrogen temperature.

The examples that treated the gas adsorption in such nanoscale dimension structures, seem to converge on one point: the possibility of adsorbing hydrogen at high rates for practical purposes. SWNTs present narrow pore size distribution and this characteristic should be favorable to high adsorptive material properties.

At room temperature it was established that hydrogen gas was efficiently adsorbed in carbon nanotube materials. By using Monte-Carlo simulations hydrogen adsorption was computed at moderate pressures and it was demonstrated that the amount of gas stored by adsorption in carbon nanotubes was larger than that stored by gas compression. The maximum of adsorption at $293 \mathrm{~K}$ was obtained with a hexagonal configuration of nanotubes distant from $0: 7 \mathrm{~nm}$ and with diameter equal to $1: 2 \mathrm{~nm}$.

The differences of adsorption amounts published are mainly attributed to:

the interaction potential models used to describe the gas-solid interaction as in the works of Wang and Johnson ;

the sampling method used in simulations ;

the adsorbent configurations (including the values of the tube diameters, the tube lengths and the inter-tube spacing) that permit the hydrogen gas either to move in a large adsorption volume or just to be con9ned in the interstitial pores. Moreover, due to the sterical effects that could occur within thin pores, like in nanomaterial ones, which are attributed to the tube arrangement within a row of nanotubes;

single- or multi-walled carbon nanotubes;

open or closed tubes which are important for the adsorption phenomenon (open tubes enable gas adsorption both inside and outside the tubes whereas closed tubes do not) and also

the reactive surface of the adsorbent material considered. Indeed, this latter value is dependent on the distance considered between consecutive tubes the so-called inter-tube spacing. Moreover, it is important to mention this possibility seem to experimentally control this parameter. Then, considering the molecular simulation works, it is noticeable that the control of microscopic parameters during the nanotube synthesis (tube diameter and length: : :) which would enable an optimization of the material structure should then enable the control of the gas adsorption process(FL Darkrim et al., 2002).

\subsection{First-principles calculations (chemisorption)}

Monte Carlo calculations based on classical potentials are not well suited to study chemical processes. However, it is likely that hydrogen and carbon react chemically. In fact, the infrared experiments of Chen et al. (P Chen et al., 1999) indicate the formation of C H bonds. In order to study this kind of storage, a more sophisticated method capable of quantummechanically treating the bond breaking and the bond formation is required. $\mathrm{Ab}$ initio calculations, capable of describing chemical processes have been performed on nanotubes by Lee et al. (S. Lee \& Lee, 2000)

Lee and Lee (S. Lee \& Lee, 2000) have performed density-functional and density-functionalbased tight binding calculations to search for hydrogen chemisorption sites and predict the maximum storage capability in single-walled carbon nanotubes (SWCNs). Their calculations are performed at zero Kelvin and show two energetically favoured configurations in which 
hydrogen atoms are chemisorbed on the nanotubes. The lowest energy one (zigzag type) is that in which the hydrogen atoms are bonded alternately outside and inside the tube (Fig. 7c). Stable but $0.56 \mathrm{eV}$ higher in energy per $\mathrm{C}-\mathrm{H}$ bond is the arch-type arrangement, shown in Fig. $7 \mathrm{~b}$. These configurations are stable in spite of the deformations induced on the $\mathrm{C}-\mathrm{C}$ bonds by the chemisorption process. The authors have also investigated the possibility of absorbing all the hydrogen atoms on the inside. It is, however, energetically favorable for the hydrogen atoms to recombine and form molecules, which are then physisorbed inside the nanotube, leading to physisorption. The maximum possible adsorption would be an apparently encouraging $14 \%$ in weight.
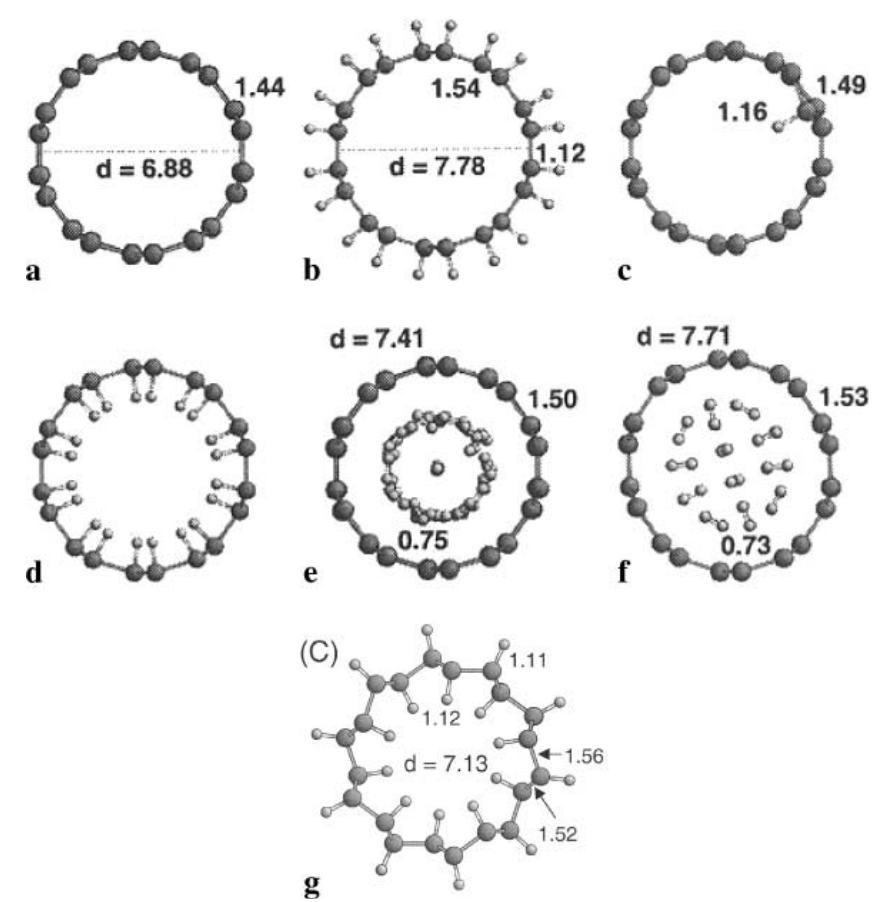

Fig. 7a-g. The calculations of Lee and Lee for chemical adsorption in singlewalled carbon nanotubes (SWCNs). a $(5,5)$ SWCN before the storage; b covalent storage outside the tube archtype $(\theta=1)$; c adsorption of a single hydrogen atom inside the tube; $d$ initial and e fully relaxed geometry of adsorbed hydrogen inside the tube walls; $\mathrm{f}$ hydrogen adsorption inside the tube with $\theta=1.2$; $\mathrm{g}$ zigzag-type geometry

In a later publication, Lee et al. (S. Lee, An, Lee, Seifert, \& Frauenheim, 2001b) addressed the issue of mechanisms for the insertion of hydrogen molecules inside the nanotubes. This is in fact a non-trivial problem even if the nanotubes are open. The very high aspect ratio makes the filling process through the open nanotubes rather inefficient. Lee et al. hypothesized a multi-step process. An hydrogen atom bonded to a carbon atom in an arch-type geometry pushes down the carbon atom and then flips into the $\mathrm{C}-\mathrm{C}$ mid-bond; the activation barrier has an energy of $1.51 \mathrm{eV}$. After the $\mathrm{H}$ atom has flipped into the capillary, the $\mathrm{C}-\mathrm{C}$ bond is 
recovered. Once the first atom has flipped in, the nearest-neighbour top site atoms can flip in more easily with the lower activation barrier of $0.74 \mathrm{eV}$, as shown in Fig. 2 II leading to a continuous flip-in process. One may also consider a zigzag flip-in process, i.e., a flip-in process for every second hydrogen (Fig. 8 III). This process has an activation barrier of 0.93 $\mathrm{eV}$ and will result in a zigzag-type geometry. Once the zigzag-type geometry, with a coverage of $\theta=1.0$, is formed, the kick-in mechanism described in Fig. 8 IV could explain hydrogen insertion in the nanotube. Repeating the kick-in process will eventually lead to the formation of molecular hydrogen inside the tube. In order to complete the storage mechanism, Lee et al. also described a similar hydrogen extraction mechanism with a low energy barrier.

Lee et al. (S. M. Lee et al., 2000) addressed the problem of storage in MWNTs. They claim that hydrogen atom chemisorption is favored between the tube walls where hydrogen storage capacity is independent of diameter, and they estimate it to be of the order of $7.7 \mathrm{wt} \%$.

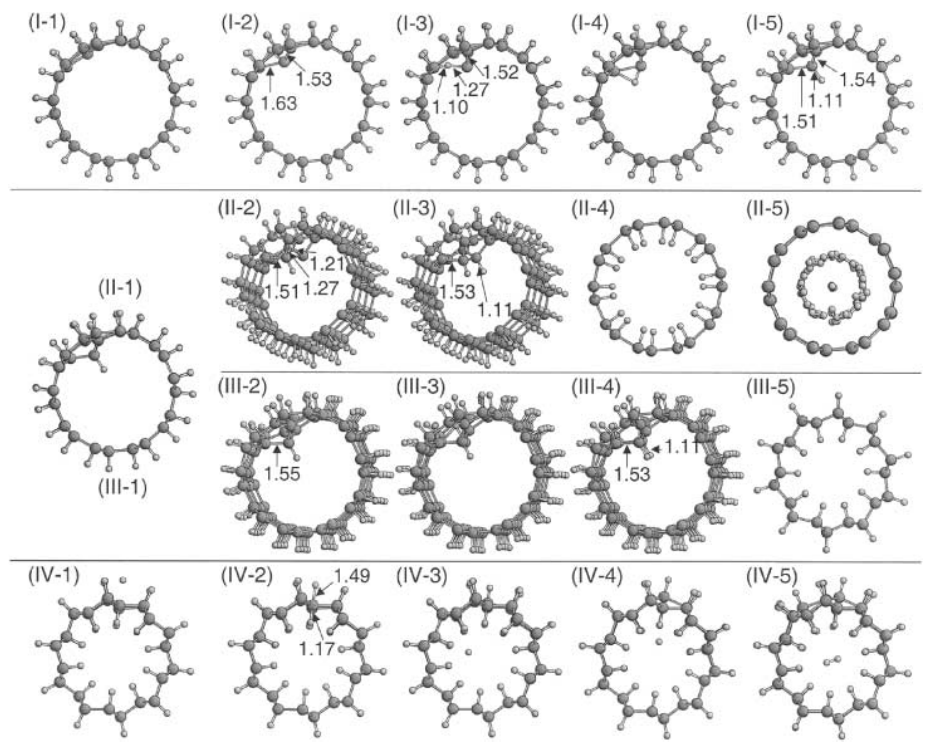

Fig. 8. The calculations of Lee et al. for possible pathways of hydrogen insertion. (I) Flip-in mechanism, (II) continuous flip-in mechanism leading to the formation of molecular hydrogen inside the tube, (III) zigzag flip-in mechanism, (IV) kick-in mechanism

Bilic' et al(Bilic \& Gale, 2008). modeled the energetics of hydrogen chemisorption on a $(6,6)$ nanotube using gradient-corrected density functional theory. The adsorption was investigated over a range of coverages, from close to the zero limit to a full monolayer. They found that the attachment of the first isolated admolecule on the tube is endothermic. However, with increasing coverage, the character of chemisorption dramatically changes to exothermic, making the dissociative addition of new $\mathrm{H}_{2}$ molecules increasingly favorable. They explored possible routes for the formation of energetically favourable fully hydrogenated nanotubes. The mechanism termed the $1-2 \mathrm{~d}$ exoaddition is predicted to have the potential of adsorbing between 1/3 and 1/2 ML of hydrogen on the outer face of the tube. The initial kinetics of such a process is predicted to proceed at an increasing rate with 
increasing degree of coverage. If a modified mechanism is considered, a combined 1-2d exo/endoaddition, full saturation of the tube is predicted, resulting in adsorption energy of $0.618 \mathrm{eV}$ per molecule and a hydrogen uptake of $7.7 \mathrm{wt} \%$. The fact that such structures are not observed experimentally is attributed to the highly activated nature of the dissociative chemisorption and the less reactive character of larger, more realistic nanotubes(Meregalli \& Parrinello, 2001).

\section{Conclusion}

The present examination of the actual state of the art of hydrogen adsorption in carbon nanotubes involves the fundamental question: is adsorption in nanotubes a reliable mode of hydrogen storage?

This work was devoted to experimental and computational studies of carbon nanotube adsorption property. These materials, if one considers some articles published in the literature, seem to be good candidates for the hydrogen storage process. However, some questions persist!! In the future, some explanations are still needed for all scientists who are interested in the hydrogen storage by adsorption in carbon nanomaterials. In particular, as concerns the surface properties of these adsorbent materials: the chemical treatment during or after the carbon nanotubes synthesis; the mechanical treatment made on the tubes in order to open their extremities and enable the gas adsorption inside the tubes; the amount of reactive nanotubes (attributed to their low purity rate after their synthesis) taken into account in the gas adsorption calculations; the presence or not of heteroatoms at their surface which can contribute to modify the electronic density at the nanotube surface and then the reactivity of the material can be reinforced (for instance it is possible to intercalate alkaline atoms between consecutive tubes); the accuracy of the volumetric and the gravimetric measurement methods should be mentioned; the definition of the hydrogen 'uptake' used by the authors in their works (excess of adsorption or total amount of the gas) . Once these respective main influencing factors on the hydrogen adsorption in carbon nanomaterials are well determined, one will be able to determine, explain, and validate the various adsorption amounts published in the literature and to control their own adsorption data which are unfortunately often obtained on their own characterized materials.

\section{References}

Adu, C., Sumanasekera, G., Pradhan, B., Romero, H. and Eklund, P. (2001). Carbon nanotubes: a thermoelectric nano-nose. Chemical Physics Letters, Vol, 337 No, 1-3, pp. 31-35

Badzian, A., Badzian, T., Breval, E. and Piotrowski, A. (2001). Nanostructured, nitrogendoped carbon materials for hydrogen storage. Thin Solid Films, Vol, 398, pp. 170-174

Bilic, A. and Gale, J. D. (2008). Chemisorption of molecular hydrogen on carbon nanotubes: A route to effective hydrogen storage? Journal of Physical Chemistry C, Vol, 112 No, 32, pp. 12568-12575,1932-7447

Bilić, A. and Gale, J. D. (2008). Chemisorption of Molecular Hydrogen on Carbon Nanotubes: A Route to Effective Hydrogen Storage? The Journal of Physical Chemistry C, Vol, 112 No, 32, pp. 12568-12575,1932-7447 
Cao, A., Zhu, H., Zhang, X., Li, X., Ruan, D., Xu, C., Wei, B., Liang, J. and Wu, D. (2001). Hydrogen storage of dense-aligned carbon nanotubes. Chemical Physics Letters, Vol, 342 No, 5-6, pp. 510-514

Chambers, A., Park, C., Baker, R. and Rodriguez, N. (1998). Hydrogen storage in graphite nanofibers. Journal of Physical Chemistry B-Condensed Phase, Vol, 102 No, 22, pp. 4253-4256

Chen, C. and Huang, C. (2007). Hydrogen storage by KOH-modified multi-walled carbon nanotubes. International Journal of Hydrogen Energy, Vol, 32 No, 2, pp. 237-246

Chen, P., Wu, X., Lin, J. and Tan, K. (1999). High H2 uptake by alkali-doped carbon nanotubes under ambient pressure and moderate temperatures. Science, Vol, 285 No, 5424, pp. 91

Chen, P., Wu, X., Lin, J. and Tan, K. L. (1999). High H2 Uptake by Alkali-Doped Carbon Nanotubes Under Ambient Pressure and Moderate Temperatures. Science, Vol, 285 No, 5424, pp. 91-93

Chen, Y., Shaw, D., Bai, X., Wang, E., Lund, C., Lu, W. and Chung, D. (2001). Hydrogen storage in aligned carbon nanotubes. Applied Physics Letters, Vol, 78, pp. 2128

Darkrim, F. and Levesque, D. (2000). High Adsorptive Property of Opened Carbon Nanotubes at 77 K. The Journal of Physical Chemistry B, Vol, 104 No, 29, pp. 67736776,1520-6106

Darkrim, F., Malbrunot, P. and Tartaglia, G. (2002). Review of hydrogen storage by adsorption in carbon nanotubes. International Journal of Hydrogen Energy, Vol, 27 No, 2, pp. 193-202

Dillon, A. and Heben, M. (2001). Hydrogen storage using carbon adsorbents: past, present and future. Applied Physics A: Materials Science E Processing, Vol, 72 No, 2, pp. 133142

Dillon, A. C., Jones, K. M., Bekkedahl, T. A., Kiang, C. H., Bethune, D. S. and Heben, M. J. (1997). Storage of hydrogen in single-walled carbon nanotubes. Nature, Vol, 386 No, 6623, pp. 377-379

Hirscher, M., Becher, M., Haluska, M., Dettlaff-Weglikowska, U., Quintel, A., Duesberg, G., Choi, Y., Downes, P., Hulman, M. and Roth, S. (2001). Hydrogen storage in sonicated carbon materials. Applied Physics A: Materials Science E Processing, Vol, 72 No, 2, pp. 129-132

Hirscher, M., Becher, M., Haluska, M., Quintel, A., Skakalova, V., Choi, Y., DettlaffWeglikowska, U., Roth, S., Stepanek, I. and Bernier, P. (2002). Hydrogen storage in carbon nanostructures. Journal of Alloys and Compounds, Vol, 330, pp. 654-658

Hwang, J., Gommans, H., Ugawa, A., Tashiro, H., Haggenmueller, R., Winey, K., Fischer, J., Tanner, D. and Rinzler, A. (2000). Polarized spectroscopy of aligned single-wall carbon nanotubes. Physical Review B, Vol, 62 No, 20, pp. 13310-13313

Kataura, H., Kumazawa, Y., Maniwa, Y., Umezu, I., Suzuki, S., Ohtsuka, Y. and Achiba, Y. (1999). Optical properties of single-wall carbon nanotubes. Synthetic Metals, Vol, 103 No, 1-3, pp. 2555-2558

Kubas, G. (2001). Metal-dihydrogen and -bond coordination: the consummate extension of the Dewar-Chatt-Duncanson model for metal-olefin bonding. Journal of Organometallic Chemistry, Vol, 635 No, 1-2, pp. 37-68 
Lee, S., An, K., Lee, Y., Seifert, G. and Frauenheim, T. (2001a). A hydrogen storage mechanism in single-walled carbon nanotubes. J. Am. Chem. Soc, Vol, 123 No, 21, pp. 5059-5063

Lee, S., An, K., Lee, Y., Seifert, G. and Frauenheim, T. (2001b). Novel mechanism of hydrogen storage in carbon nanotubes. JOURNAL-KOREAN PHYSICAL SOCIETY, Vol, 38 No, 6, pp. 686-691

Lee, S. and Lee, Y. (2000). Hydrogen storage in single-walled carbon nanotubes. Applied Physics Letters, Vol, 76, pp. 2877

Lee, S. M., Park, K. S., Choi, Y. C., Park, Y. S., Bok, J. M., Bae, D. J., Nahm, K. S., Choi, Y. G., Yu, S. C., Kim, N.-g., Frauenheim, T. and Lee, Y. H. (2000). Hydrogen adsorption and storage in carbon nanotubes. Synthetic Metals, Vol, 113 No, 3, pp. 209-216,03796779

Liu, C., Fan, Y., Liu, M., Cong, H., Cheng, H. and Dresselhaus, M. (1999). Hydrogen storage in single-walled carbon nanotubes at room temperature. Science, Vol, 286 No, 5442, pp. 1127

Liu, C., Fan, Y. Y., Liu, M., Cong, H. T., Cheng, H. M. and Dresselhaus, M. S. (1999). Hydrogen Storage in Single-Walled Carbon Nanotubes at Room Temperature. Science, Vol, 286 No, 5442, pp. 1127-1129

Liu, C., Yang, Q., Tong, Y., Cong, H. and Cheng, H. (2002). Volumetric hydrogen storage in single-walled carbon nanotubes. Applied Physics Letters, Vol, 80, pp. 2389

Meregalli, V. and Parrinello, M. (2001). Review of theoretical calculations of hydrogen storage in carbon-based materials. Applied Physics A: Materials Science E Processing, Vol, 72 No, 2, pp. 143-146

Nützenadel, C., Züttel, A., Chartouni, D. and Schlapbach, L. (1999). Electrochemical storage of hydrogen in nanotube materials. Electrochemical and solid-state letters, Vol, 2, pp. 30

Nijkamp, M., Raaymakers, J., Van Dillen, A. and De Jong, K. (2001). Hydrogen storage using physisorption-materials demands. Applied Physics A: Materials Science E Processing, Vol, 72 No, 5, pp. 619-623

Nishimiya, N., Ishigaki, K., Takikawa, H., Ikeda, M., Hibi, Y., Sakakibara, T., Matsumoto, A. and Tsutsumi, K. (2002). Hydrogen sorption by single-walled carbon nanotubes prepared by a torch arc method. Journal of Alloys and Compounds, Vol, 339 No, 1-2, pp. 275-282

Pinkerton, F., Wicke, B., Olk, C., Tibbetts, G., Meisner, G., Meyer, M. and Herbst, J. (2000). Thermogravimetric measurement of hydrogen absorption in alkali-modified carbon materials. J. Phys. Chem. B, Vol, 104 No, 40, pp. 9460-9467

Popov, V. (2004). Carbon nanotubes: properties and application. Materials Science $\mathcal{E}$ Engineering R, Vol, 43 No, 3, pp. 61-102

Pradhan, B., Harutyunyan, A., Stojkovic, D., Grossman, J., Zhang, P., Cole, M., Crespi, V., Goto, H., Fujiwara, J. and Eklund, P. (2002). Large cryogenic storage of hydrogen in carbon nanotubes at low pressures. Journal of Materials Research, Vol, 17 No, 9, pp. 2209-2216

Ritschel, M., Uhlemann, M., Gutfleisch, O., Leonhardt, A., Graff, A., Täschner, C. and Fink, J. (2002). Hydrogen storage in different carbon nanostructures. Applied Physics Letters, Vol, 80, pp. 2985 
Sakintuna, B., Lamari-Darkrim, F. and Hirscher, M. (2007). Metal hydride materials for solid hydrogen storage: a review. International Journal of Hydrogen Energy, Vol, 32 No, 9 , pp. 1121-1140

Schulz, R., Huot, J., Liang, G., Boily, S., Lalande, G., Denis, M. and Dodelet, J. (1999). Recent developments in the applications of nanocrystalline materials to hydrogen technologies. Materials science $\mathcal{E}$ engineering. A, Structural materials: properties, microstructure and processing, Vol, 267 No, 2, pp. 240-245

Smith Jr, M., Bittner, E., Shi, W., Johnson, J. and Bockrath, B. (2003). Chemical activation of single-walled carbon nanotubes for hydrogen adsorption. J. Phys. Chem. B, Vol, 107 No, 16, pp. 3752-3760

Ströbel, R., Garche, J., Moseley, P., Jörissen, L. and Wolf, G. (2006). Hydrogen storage by carbon materials. Journal of Power Sources, Vol, 159 No, 2, pp. 781-801

Ströbel, R., Garche, J., Moseley, P. T., Jörissen, L. and Wolf, G. (2006). Hydrogen storage by carbon materials. Journal of Power Sources, Vol, 159 No, 2, pp. 781-801,0378-7753

Tibbetts, G., Meisner, G. and Olk, C. (2001). Hydrogen storage capacity of carbon nanotubes, filaments, and vapor-grown fibers. Carbon, Vol, 39 No, 15, pp. 2291-2301

Wang, Q. and Johnson, J. (1999). Molecular simulation of hydrogen adsorption in singlewalled carbon nanotubes and idealized carbon slit pores. The Journal of Chemical Physics, Vol, 110, pp. 577

Wu, X., Chen, P., Lin, J. and Tan, K. (2000). Hydrogen uptake by carbon nanotubes. International Journal of Hydrogen Energy, Vol, 25 No, 3, pp. 261-265

Yang, R. (2000). Hydrogen storage by alkali-doped carbon nanotubes-revisited. Carbon, Vol, 38 No, 4, pp. 623-626

Ye, Y., Ahn, C., Witham, C., Fultz, B., Liu, J., Rinzler, A., Colbert, D., Smith, K. and Smalley, R. (1999). Hydrogen adsorption and cohesive energy of single-walled carbon nanotubes. Applied Physics Letters, Vol, 74, pp. 2307

Ye, Y., Ahn, C. C., Witham, C., Fultz, B., Liu, J., Rinzler, A. G., Colbert, D., Smith, K. A. and Smalley, R. E. (1999). Hydrogen adsorption and cohesive energy of single-walled carbon nanotubes. Applied Physics Letters, Vol, 74 No, 16, pp. 2307-2309

Züttel, A. (2003). Materials for hydrogen storage. Materials today, Vol, 6 No, 9, pp. 24-33

Zhu, H., Cao, A., Li, X., Xu, C., Mao, Z., Ruan, D., Liang, J. and Wu, D. (2001). Hydrogen adsorption in bundles of well-aligned carbon nanotubes at room temperature. Applied Surface Science, Vol, 178 No, 1-4, pp. 50-55 


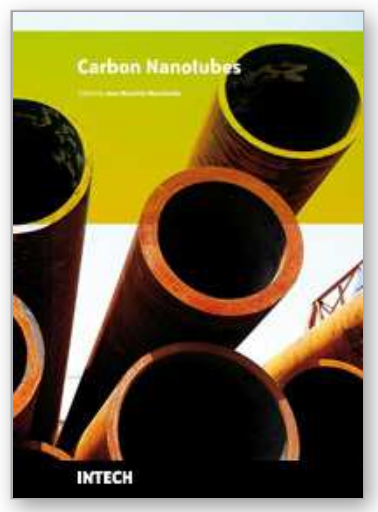

\author{
Carbon Nanotubes \\ Edited by Jose Mauricio Marulanda
}

ISBN 978-953-307-054-4

Hard cover, 766 pages

Publisher InTech

Published online 01, March, 2010

Published in print edition March, 2010

This book has been outlined as follows: A review on the literature and increasing research interests in the field of carbon nanotubes. Fabrication techniques followed by an analysis on the physical properties of carbon nanotubes. The device physics of implemented carbon nanotubes applications along with proposed models in an effort to describe their behavior in circuits and interconnects. And ultimately, the book pursues a significant amount of work in applications of carbon nanotubes in sensors, nanoparticles and nanostructures, and biotechnology. Readers of this book should have a strong background on physical electronics and semiconductor device physics. Philanthropists and readers with strong background in quantum transport physics and semiconductors materials could definitely benefit from the results presented in the chapters of this book. Especially, those with research interests in the areas of nanoparticles and nanotechnology.

\title{
How to reference
}

In order to correctly reference this scholarly work, feel free to copy and paste the following:

Yunjin Yao (2010). Hydrogen Storage Using Carbon Nanotubes, Carbon Nanotubes, Jose Mauricio Marulanda (Ed.), ISBN: 978-953-307-054-4, InTech, Available from: http://www.intechopen.com/books/carbonnanotubes/hydrogen-storage-using-carbon-nanotubes

\section{INTECH}

open science | open minds

\section{InTech Europe}

University Campus STeP Ri

Slavka Krautzeka 83/A

51000 Rijeka, Croatia

Phone: +385 (51) 770447

Fax: +385 (51) 686166

www.intechopen.com

\section{InTech China}

Unit 405, Office Block, Hotel Equatorial Shanghai

No.65, Yan An Road (West), Shanghai, 200040, China

中国上海市延安西路65号上海国际贵都大饭店办公楼405单元

Phone: +86-21-62489820

Fax: +86-21-62489821 
(C) 2010 The Author(s). Licensee IntechOpen. This chapter is distributed under the terms of the Creative Commons Attribution-NonCommercialShareAlike-3.0 License, which permits use, distribution and reproduction for non-commercial purposes, provided the original is properly cited and derivative works building on this content are distributed under the same license. 